\title{
Gap junctions in the ovary of Drosophila melanogaster: localization of innexins I, 2, 3 and 4 and evidence for intercellular communication via innexin- 2 containing channels Johannes Bohrmann* and Jennifer Zimmermann
}

\author{
Address: RWTH Aachen University, Institut für Biologie II, Abt. Zoologie und Humanbiologie, Kopernikusstrasse 16, 52056 Aachen, Germany \\ Email: Johannes Bohrmann* - bohrmann@bio2.rwth-aachen.de; Jennifer Zimmermann - jennifer.zimmermann@post.rwth-aachen.de \\ * Corresponding author
}

Published: 27 November 2008

BMC Developmental Biology 2008, 8: III doi:10.1 186/147I-213X-8-III

Received: 8 June 2008

Accepted: 27 November 2008

This article is available from: http://www.biomedcentral.com/I47I-2/3X/8/III

(c) 2008 Bohrmann and Zimmermann; licensee BioMed Central Ltd.

This is an Open Access article distributed under the terms of the Creative Commons Attribution License (http://creativecommons.org/licenses/by/2.0), which permits unrestricted use, distribution, and reproduction in any medium, provided the original work is properly cited.

\begin{abstract}
Background: In the Drosophila ovary, germ-line and soma cells are interconnected via gap junctions. The main gap-junction proteins in invertebrates are members of the innexin family. In order to reveal the role that innexins play in cell-cell communication during oogenesis, we investigated the localization of innexins I, 2, 3 and 4 using immunohistochemistry, and analyzed follicle development following channel blockade.

Results: We found innexin I predominantly localized to the baso-lateral domain of follicle cells, whereas innexin 2 is positioned apico-laterally as well as apically between follicle cells and germline cells. Innexin 3 was observed laterally in follicle cells and also in nurse cells, and innexin 4 was detected in the oolemma up to stage 8 and in nurse-cell membranes up to stage 12 . In order to test whether innexins form channels suitable for intercellular communication, we microinjected innexin antibodies in combination with a fluorescent tracer into the oocyte of stage- 10 follicles. We found that dye-coupling between oocyte and follicle cells was largely reduced by innexin- 2 antibodies directed against the intracellular $\mathrm{C}$-terminus as well as against the intracellular loop. Analyzing in vitro, between stages 10 and 14, the developmental capacities of follicles following microinjections of innexin-2 antibodies revealed defects in follicle-cell differentiation, nurse-cell regression, oocyte growth and choriogenesis.
\end{abstract}

Conclusion: Our results suggest that all analyzed innexins are involved in the formation of gap junctions in the ovary. While innexins 2 and 3 are colocalized between soma cells, innexins 2 and 4 are colocalized between soma and germ-line cells. Innexin 2 is participating in cell-cell communication via hemichannels residing in the oolemma. It is obvious that gap-junctional communication between germ-line and soma cells is essential for several processes during oogenesis.

\section{Background}

During Drosophila oogenesis, germ-line and soma cells are known to interact intimately with each other, for example concerning follicle organization, vitellogenesis and egg- shell production [1-3]. In many cases, however, the exact routes of information exchange have remained elusive. During establishment of the dorsoventral and anteroposterior axes, intercellular communication between germ- 
line and soma cells has been shown to be essential as well. Besides other mechanisms, e.g. contact of membrane receptors and their ligands [4-6], the transfer of small molecules via gap junctions might be involved in such communication processes.

In recent years, gap-junction mediated cell-cell communication in Drosophila has gained growing attention, as several mutants have been described in which specific developmental defects are correlated with altered gapjunction plaques or channels [7-14]. In arthropods, the coordination of physiological and developmental processes by means of gap-junctional communication [15-19] might be even more important than in vertebrates, since the channels of arthropods allow the exchange of molecules of up to $3000 \mathrm{D}$ [20]. In special cases, even much larger molecules, e.g. calmodulin, seem to be able to pass through gap junctions [21,22]. Recently, it has been reported that gene expression can be regulated by neighbouring cells through the exchange of siRNAs via gap junctions [23].

While vertebrates use connexins for the assembly of gapjunction channels (connexons) [24,25], the main gapjunction proteins found in invertebrates are members of the innexin family [26-29]. However, additional proteins have been detected: the innexin-related pannexins [30] and the ductins [31-33]. Neither innexins nor pannexins have homologies with either connexins or ductins. While pannexins were found only in vertebrates, ductins were observed in gap junctions of vertebrates as well as invertebrates. In some studies on insect gap junctions, further proteins have been deteced [26].

The ovarian follicle of Drosophila consists of a group of 16 germ-line cells surrounded by a layer of somatic follicle cells (Fig. 1A). The oocyte and its 15 nurse cells form a cytoplasmic continuum via intercellular bridges as well as via gap junctions, and the same holds true for the follicle cells. With the germ-line cells, however, the follicle cells are only connected via gap junctions, that have been found to vary in structure and size during the course of oogenesis $[34,35]$. By way of the intercellular distribution of microinjected fluorescent tracers, we have previously revealed stage-specific communication between oocyte and follicle cells. A variety of treatments has been found to either inhibit or to stimulate dye-coupling, e.g. acidic $\mathrm{pH}$, high intracellular $\left[\mathrm{Ca}^{2+}\right]$, octanol, dinitrophenol, a juvenile hormone analogue or 20-hydroxyecdysone [36].

Of the eight known Drosophila innexins, the mRNAs of innexins 1, 2, 3, 4 and 7 were detected in the ovary [37]. So far, only for innexin 4 the localization and functioning of the protein during early oogenesis have been analyzed
$[13,38]$. We investigated by immunoblots and immunohistochemistry the occurrence and detailed localization of innexins 1, 2, 3 and 4 throughout oogenesis. Our results suggest that all analyzed innexins are involved in the formation of ovarian gap junctions.

In order to test whether innexins form intercellular communication channels in the ovary, we microinjected various innexin antisera in combination with the fluorescent tracer Lucifer Yellow into the oocyte of stage-10 follicles. We found that only innexin-2 antibodies were able to significantly reduce the dye-coupling observed between oocyte and follicle cells. Therefore, innexin 2 is involved in cell-cell communication between germ-line and soma cells by participating in gap-junction hemichannels residing in the oolemma.

We further analyzed the developmental capacities of follicles following microinjections of innexin-2 antibodies. Since oogenesis in vitro was affected in a significant fraction of microinjected follicles, we conclude that cell-cell communication via innexin-2-containing gap junctions is essential for various processes of normal follicle development through stages 10 to 14 .

\section{Methods \\ Antisera}

For immunocytochemical and immunoblot analysis as well as for microinjections, we used the following antipeptide sera, which have been raised against cytoplasmic regions (c.f. Fig. 1B) of different innexins from Drosophila melanogaster: (1) a rabbit antiserum to the C-terminus of innexin 1 (AInx1Rb-CT) [14,39], (2) two rabbit antisera to different regions of the C-terminus of innexin 2 (AInx2Rb-CT(KLRH) and AInx2Rb-CT(REM) [14,39]), (3) a guinea-pig antiserum to the cytoplasmic loop of innexin 2 (AInx2GP-CL, R. Bauer, pers. com.), (4) a guinea-pig antiserum to the C-terminus of innexin 3 (AInx3GP-CT, R. Bauer, pers. com.), (5) a rabbit antiserum to the cytoplasmic loop of innexin 3 (AInx3Rb-CL) [40], (6) a rabbit antiserum to the C-terminus of innexin 4 (AInx4Rb-CT) $[13,38]$, and (7) as a control, a rabbit non-immune serum (NIS). The antisera to innexins 1, 2 and 3 were kindly provided by R. Bauer and M. Hoch (Bonn, Germany), whilst the antiserum to innexin 4 was a gift from S. Tazuke (Philadelphia, PA, USA).

\section{Preparation of follicles}

Drosophila melanogaster wild-type Oregon $\mathrm{R}$ flies were reared at about $20^{\circ} \mathrm{C}$ on standard food with additional fresh yeast. Individual females of 2-3 days old were killed by crushing the thorax with tweezers without previous etherization or chilling. For detailed analysis, follicles of various stages [41] were dissected out of ovaries in Drosophila PBS (DPBS) [42,43] using tungsten needles. 

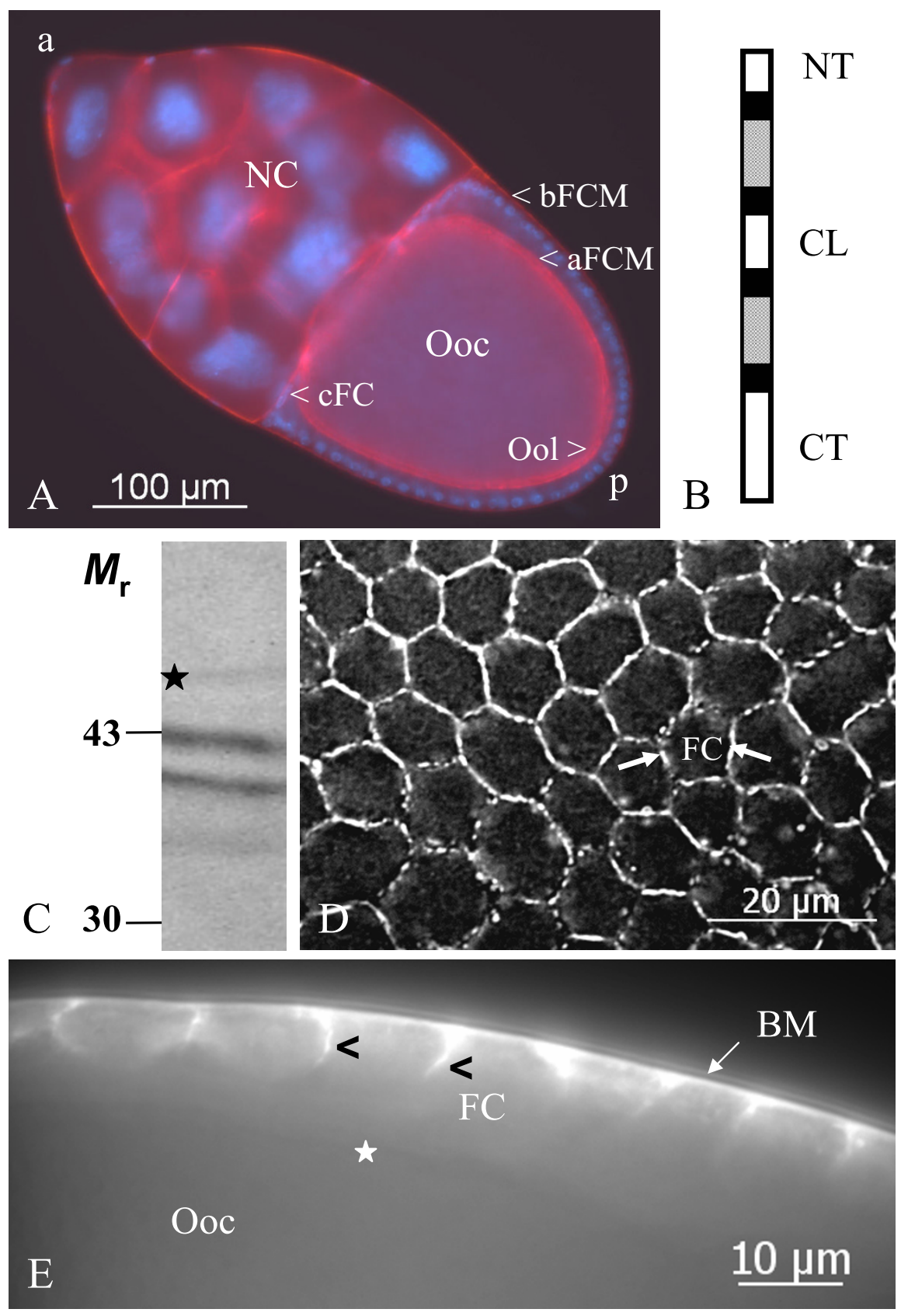

\section{Figure I}

InxI is localized to baso-lateral follicle-cell membranes. A: Drosophila stage- I0b follicle, showing different cell types and membrane regions relevant for the present study (blue: nuclei, stained with DAPI; red: F-actin, stained with rhodaminylphalloidin). B: Scheme of the predicted protein structure of innexins (white: intracellular domains (NT, N-terminus; CL, cytopasmic loop; CT, C-terminus), black: transmembrane domains, grey: extracellular domains). Hemichannels made of six molecules can be either homo- or heteromeric, channels made of two hemichannels either homo- or heterotypic. C: On immunoblots of ovary extracts using $\ln x \mid$-antiserum (AlnxIRb-CT) a weak band at the calculated molecular mass of $46 \mathrm{kDa}$ (asterisk) and two bands at 43 and $4 I \mathrm{kDa}$ are recognized. D: InxI is detected in a continuous pattern as well as in presumed gap-junction plaques at the lateral follicle-cell membranes (FC, arrows, stage I0a, surface view, WFF). E: InxI is restricted to the baso-lateral domain (arrowheads) of the follicle cells, i. e. the apical domain and the oolemma (asterisk) is not stained (stage I0a, optical median section, WFF). a, anterior; aFCM, apical follicle-cell membrane; bFCM, basal follicle-cell membrane; BM, basement membrane; cFC, centripetally migrating follicle cells; NC, nurse cells; Ooc, oocyte; Ool, oolemma. 


\section{Immunoblotting}

Immunoblots were performed as described previously [31]. In short, homogenates of ovaries were sonicated and briefly boiled. The proteins were separated using $12 \%$ SDS-PAGE and transferred to nitrocellulose membranes. Nonspecific binding sites were blocked with 5\% skimmed milk powder/PBS and the blots were incubated (1) in 1\% BSA/PBS (control), (2) in AInx1Rb-CT (diluted 1:400 with 1\% BSA/PBS), (3) in AInx2Rb-CT(KLRH, diluted 1:400), (4) in AInx2GP-CL (diluted 1:200), (5) in AInx3GP-CT (diluted 1:200), (6) in AInx3Rb-CL (diluted 1:400), and (7) in AInx4Rb-CT (diluted 1:1000), respectively. Subsequently, the appropriate biotinylated secondary antibodies (goat-anti-rabbit IgG or goat-anti-guineapig IgG, Jackson, PA, USA; diluted 1:1000), streptavidinperoxidase (Dianova, Germany; diluted 1:1000) and $\mathrm{H}_{2} \mathrm{O}_{2}$ /4-chloro-1-naphthol (Sigma, Germany) were applied, and photographs were taken using a digital camera. Each experiment was performed at least three times.

\section{Indirect immunofluorescence preparations}

For immunostaining, follicles were fixed for 30 minutes at $4{ }^{\circ} \mathrm{C}$ in $4 \%$ formaldehyde dissolved in PBS, washed in PBS and blocked for 1 hour at $20^{\circ} \mathrm{C}$ with $2 \%$ BSA/0.1\% Triton $\mathrm{X}-100 / \mathrm{PBS}$. Thereafter, the follicles were incubated overnight at $4{ }^{\circ} \mathrm{C}(1)$ in $0.5 \%$ BSA/0.1\% Triton $\mathrm{X}-100 / \mathrm{PBS}$ (control), (2) in NIS diluted 1:200 with 0.5\% BSA/0.1\% Triton X-100/PBS (control), (3) in AInx1Rb-CT (diluted 1:50), (4) in AInx2Rb-CT(REM, diluted 1:50), (5) in AInx2GP-CL (diluted 1:20), (6) in AInx3GP-CT (diluted $1: 20)$, (7) in AInx3Rb-CL (diluted 1:50), and (8) in AInx4Rb-CT (diluted 1:4000), respectively.

After washing 6 times for $10 \mathrm{~min}$, the follicles were either treated with a 1:100 dilution of biotinylated goat-antirabbit IgG or with a 1:100 dilution of biotinylated goatanti-guinea-pig IgG (Jackson) for $1 \mathrm{~h}$ at $20^{\circ} \mathrm{C}$, washed again 6 times and incubated in a 1:1000 dilution of streptavidin-Texas Red (Dianova) for $30 \mathrm{~min}$. For doublestaining experiments, a 1:2000 dilution of goat-anti-rabbit-Cy3 (Jackson) as well as a 1:100 dilution of donkeyanti-guinea-pig-FP488 (FluoProbes, Interchim, France) were used for $1 \mathrm{~h}$ at $20^{\circ} \mathrm{C}$. Washing was repeated 6 times, and the nuclei were stained with $0.2 \mu \mathrm{g} / \mathrm{ml}$ DAPI (Sigma) in PBS for $3 \mathrm{~min}$. Thereafter, the follicles were either mounted in Fluoromount G (Interchim) and viewed in a Zeiss Axiovert 200 wide-field fluorescence (WFF) microscope equipped with a Hamamatsu Orca ER camera, or they were mounted in glycerine/PBS 1:1 and viewed in a Leica DMRE laser-scanning microscope (LSM). Each experiment was performed at least three times.

\section{Microinjection procedure and analysis of dye-coupling}

Stage-10 follicles, in which the oocyte occupies about $1 / 2$ of the follicle's volume, were carefully isolated in R-14 medium $[42,43]$. Samples of 5-10 follicles lacking any signs of injury were washed in R-14 and immediately transferred to the microinjection chamber. The microinjection procedure was described previously [36,44]. In short, micropipettes were pulled from 1-mm glass capillaries containing a filament, and microinjections were carried out on an inverted epifluorescence microscope (Zeiss Axiovert 200). The injection pipette (tip diameter 1-2 $\mu \mathrm{m}$ ) was mounted on a motorized micromanipulator equipped with a piezo translator (Märzhäuser, PM 20), and coupled to a microinjector (NPI, PDES-O2T). The recipient follicle was held in place with a suction pipette (tip diameter 30-50 $\mu \mathrm{m}$ ) mounted on a second micromanipulator, and coupled to a screw-adjustable syringe. The volume injected into each oocyte (using a pressure of about $300 \mathrm{hPa}$ ) was in the order of $100 \mathrm{pl}$, which is equivalent to about $5 \%$ of the oocyte volume [45].

In control experiments, the fluorescent tracer Lucifer Yellow CH (LY, Sigma; 2.5\% solution (w/v) in distilled water) and NIS (diluted 1:5 with distilled water and mixed 1:1 with 5\% LY-solution), respectively, were used. In further experiments the following antisera (mixed 1:1 with 5\% LY-solution) were microinjected: (1) AInx1RbCT, (2) AInx2Rb-CT(KLRH), (3) AInx2Rb-CT(REM)), (4) AInx2GP-CL, (5) AInx3GP-CT, (6) AInx3Rb-CL, and (7) AInx4Rb-CT, respectively. Using the Hamamatsu Orca ER camera, very weak levels of fluorescence were detectable in the follicular epithelium. In Table 1, the results of six experiments were pooled. Statistical significance of differences between relative frequencies of dye-couplig was established at the $\alpha=0.05$ level using the $X^{2}$-test.

\section{In-vitro development of microinjected follicles}

Microinjections were carried out as described above. Invitro culture of follicles through stages $10 \mathrm{~b}$ to 14 was performed in small alcohol-cleansed glass blocks containing $100 \mu \mathrm{l}$ R-14 medium covered by an air volume of about 1 $\mathrm{ml}$ and a glass slide at $20^{\circ} \mathrm{C}$ in a wet chamber $[43,45]$. In order to test for the effects of gap-junctional blockade on follicle development, the antisera AInx2Rb-CT(KLRH) and AInx2GP-CL were microinjected into the oocyte. Noninjected follicles, follicles injected with distilled water, follicles injected with NIS and follicles injected with AInx4RbCT served as controls. Photographs were taken following microinjections at stage $10 \mathrm{~b}$ and after 8 hours using a Nikon SMZ1000 stereomicroscope equipped with a Canon digital camera. Staging of follicles developed in vitro was performed as described before [45]. In Table 2, the results of four experiments were pooled. For statistical evaluation the $X^{2}$-test $(\alpha=0.05)$ was used.

\section{Results \\ Detection of innexins in the ovary by immunoblotting}

Using immunoblots, we analyzed whether the mRNAs of innexins 1, 2, 3 and 4, which have been described in the Drosophila ovary [37], become translated during oogene- 
Table I: Summary of experiments analyzing dye-coupling between oocyte and follicle cells

\begin{tabular}{lll}
\hline Microinjected solution & $n$ injected for dye-coupling & \% follicles with dye-coupling \\
\hline $\mathrm{H}_{2} \mathrm{O}$ dest. & 13 & 85 \\
$\mathrm{NIS}$ & 40 & 48 \\
AlnxIRb-CT & 34 & 50 \\
Alnx2Rb-CT(KLRH) & 39 & $17^{*}$ \\
Alnx2Rb-CT(REM) & 39 & 38 \\
Alnx2GP-CL & 53 & $30^{*}$ \\
Alnx3GP-CT & 38 & 37 \\
Alnx3Rb-CL & 20 & 40 \\
Alnx4Rb-CT & 22 & 46
\end{tabular}

*, significantly different from NIS $(P<0.05)$; $n$, number of injected stage-10 follicles; for further details, see text

sis. In ovarian extracts, some of the used innexin antisera, that have all been characterized previously ([13,14,38-40] and R. Bauer, pers. com.), revealed only weak bands at the molecular masses calculated from the amino-acid sequences of the proteins. But further bands at lower molecular masses were always detected, which are specific for the respective innexin (Inx), and which have been observed in embryonic extracts too (not shown).

The antiserum AInx1Rb-CT against the C-terminus of Inx1 (for different protein domains, see Fig. 1B) recognized a weak band at the calculateded molecular mass of $46 \mathrm{kDa}$ (Fig. 1C; also R. Bauer, pers. com.). Two Inx2-antisera, AInx2Rb-CT(KLRH) against the C-terminus and AInx2GP-CL against the cytoplasmic loop, both recognized bands at the calculateded molecular mass of $42 \mathrm{kDa}$ (Fig. 2A, A'; also [14]), whereas the reaction of AInx2RbCT(REM) was very weak (not shown; also R. Bauer, pers. com.). Both Inx3-antisera, AInx3GP-CT against the C-terminus and AInx3Rb-CL against the cytoplasmic loop, recognized bands at the calculateded molecular mass of 45 kDa (Fig. 3A, A'; also [40]). The antiserum AInx4Rb-CT against the C-terminus of Inx 4 recognized a very weak band at the calculateded molecular mass of $43 \mathrm{kDa}$ (not shown; also $[13,38])$.

\section{Localization of innexins during oogenesis by immunocytochemistry}

Using indirect immunofluorescence microscopy, we investigated the cellular localization of innexins 1, 2, 3 and 4 during the course of Drosophila oogenesis. For orientation, an overview of a stage-10b follicle is shown in Fig. 1A. All antisera clearly recognized cytoplasmic as well as membranous antigens, with the exception of AInx2RbCT(KLRH), which appeared to be less suitable for wholemount immunocytochemistry (but was optimal for SL2 cells; R. Bauer, pers. com.). Punctate membrane labeling is presumed to represent gap-junction plaques, while diffuse or continuous membrane labeling represents either homogeniously dispersed channels or hemichannels. Labeling of cytoplasmic clouds or particles is presumed to represent channel precursors. The results presented below are specific, since in control preparations, incubated without primary antibodies, no staining was observed (e.g. Fig. 2C).

We found Inx1 predominantly localized to the baso-lateral domain of follicle cells (Fig. 1D, E), whereas in germline cells no membrane labeling was detected. Inx2, on the other hand, is positioned apico-laterally between follicle cells as well as apically between follicle cells and oocyte and also between follicle cells and nurse cells (Fig. 2B-F). Inx2-labeling is present in the oolemma (Figs. 2F, 4D) and in the nurse-cell membranes (Figs. 2F, 3E). During stages 10 and 11, when the apical follicle-cell membranes are connected with the oolemma via gap junctions located on microvilli spanning the developing vitelline membrane [41], Inx2-plaques are found in this region (Fig. 2B-D). In the nurse cells, the distribution of Inx2 changed stage-specifically, starting with diffuse localiza-

Table 2: Summary of experiments analyzing in-vitro development

\begin{tabular}{lll}
\hline Microinjected solution & $n$ injected for in-vitro development & \% follicles developed to stages I2-14 \\
\hline n. i. & 8 & 88 \\
$\mathrm{H}_{2} \mathrm{O}$ dest. & 45 & 70 \\
$\mathrm{NIS}$ & 20 & 55 \\
$\mathrm{Aln} \times 2 \mathrm{Rb}-\mathrm{CT}(\mathrm{KLRH})$ & 50 & $24^{*}$ \\
Alnx2GP-CL & $\mathrm{II}$ & $27^{*}$ \\
Alnx4Rb-CT & 14 & 50 \\
\hline
\end{tabular}

*, significantly different from NIS $(P<0.05)$; $n$, number of injected stage- I Ob follicles; for further details, see text 

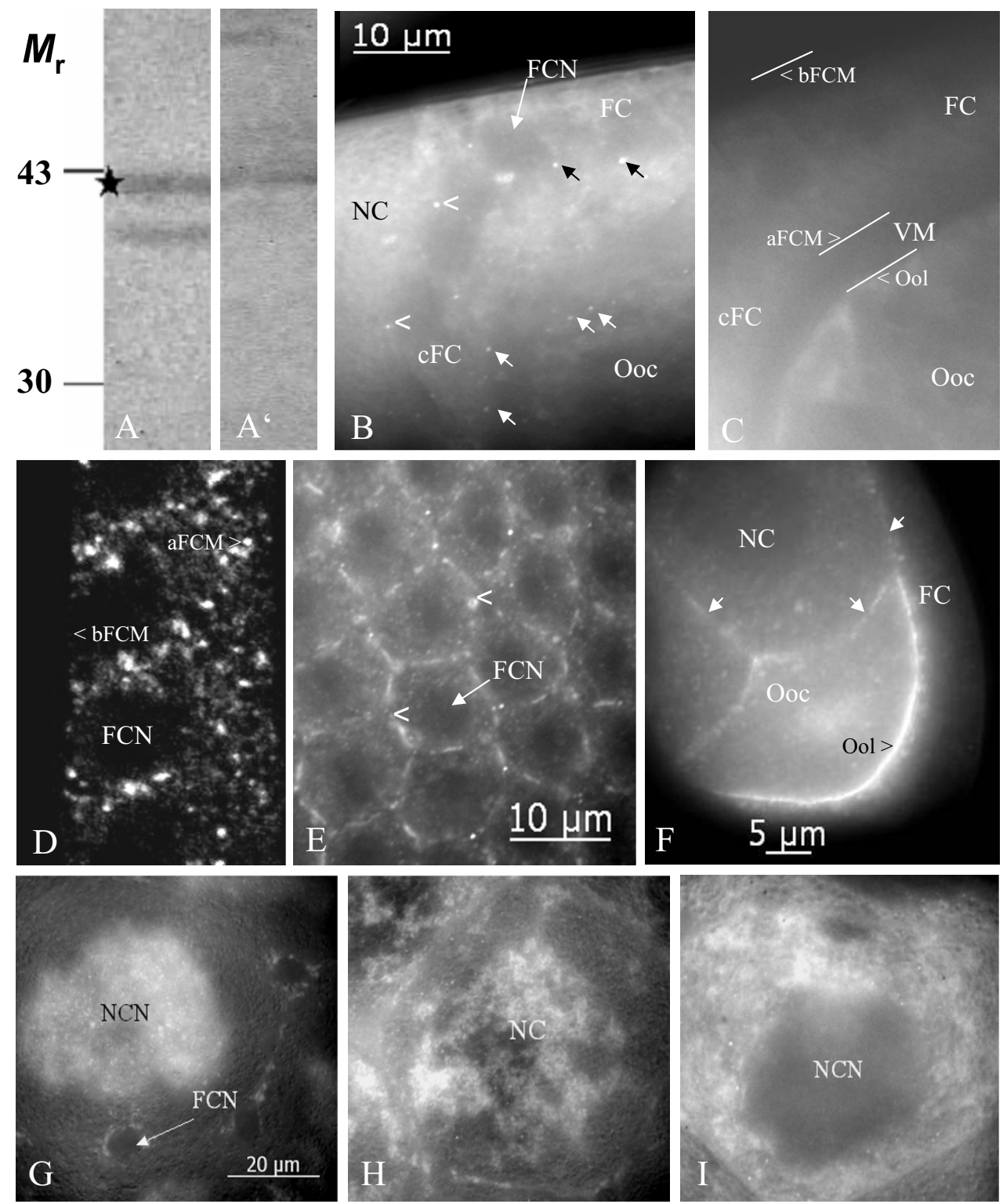

\section{Figure 2}

Inx2 is localized to membrane plaques in follicle cells and germ-line cells and is distributed stage-specifically in nurse cells. On immunoblots of ovary extracts using $\ln x 2$-antisera (A: Alnx2Rb-CT(KLRH), $\left.A^{\prime}: A \ln \times 2 G P-C L\right)$ a band at the calculated molecular mass of $42 \mathrm{kDa}$ (asterisk) and a band at $39 \mathrm{kDa}$ are recognized. $\mathrm{B}$ : Inx2 is localized to presumed gap-junction plaques between follicle cells as well as between follicle cells and germ-line cells (stage IOb, Alnx2Rb-CT(REM), optical median section, WFF; see also Fig. 4D). Black arrows point to apico-laterally situated plaques between follicle cells, white arrows point to plaques between follicle cells and oocyte, and arrowheads point to plaques between centripetally migrating follicle cells and nurse cells. C: Control follicle (NIS) showing the anterior-dorsal region of the oocyte and the follicular epithelium (comparable to the region shown in B). Different membrane regions are marked with white lines. Apical follicle-cell membranes make contact with the oolemma via microvilli spanning the developing vitelline membrane (VM). D, E: Inx2 is found in lateral and apical, but not in basal follicle-cell membranes (arrowheads, stage II, Anti-Inx2GP-CL, D: LSM, E: optical tangential section, WFF). F: Inx 2 is located in the oolemma (arrowhead) as well as in nurse-cell membranes (white arrows, stage 8 , Anti-Inx2GP-CL, optical median section, WFF). G-I: Stage-specific distribution of $\ln \times 2$ in the nurse cells (Alnx2Rb-CT(REM), WFF): During stage I0a (G), Inx2 accumulates around nurse-cell nuclei (NCN). During stage $10 \mathrm{~b}(\mathrm{H})$, Inx2 becomes dispersed in the cytoplasm. During nurse-cell regression (stage $\mathrm{II}, \mathrm{I}$ ), Inx2 is found in cytoplasmic clouds and in particles that become transported into the oocyte. FCN, follicle-cell nucleus (not stained); for further abbreviations, see legend to Fig. I. 

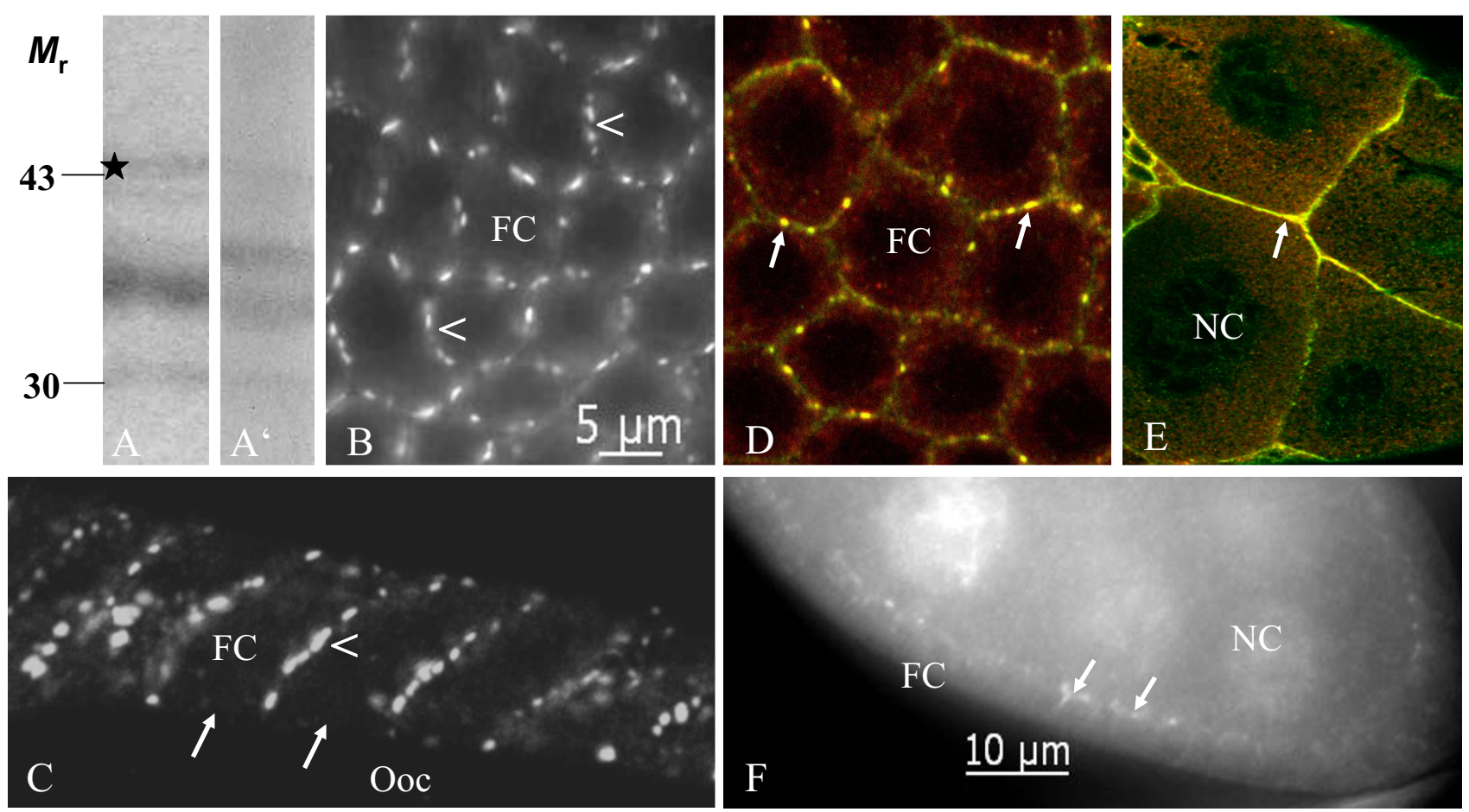

Figure 3

Inx3 is colocalized with Inx2 to membrane plaques in follicle cells and nurse cells. On immunoblots of ovary extracts using Inx3-antisera (A: Alnx3GP-CT, A': Alnx3Rb-CL) a weak band at the calculated molecular mass of $45 \mathrm{kDa}$ (asterisk) and two bands at 35 and $30 \mathrm{kDa}$ are recognized. $\mathrm{B}, \mathrm{C}$ : Inx3 is localized to presumed gap-junction plaques between follicle cells (stage IOa). It is expressed in a continuous as well as punctate pattern at the lateral membranes (arrowheads, B: Alnx3RbCL, WFF, C: Alnx3GP-CT, LSM), but is missing at the apical membranes and at the oolemma (arrows). D, E: Inx2 (red, Alnx2Rb-CT(REM)) and Inx3 (green, Alnx3GP-CT) are colocalized in lateral follicle-cell membranes (yellow, arrows in D, stage IOa, LSM) as well as in nurse-cell membranes (yellow, arrow in E, stage IOb, LSM). F: In young follicles, Inx3 is located in plaques between nurse cells and follicle cells (arrows) and around nurse-cell nuclei (stage 7, Alnx3Rb-CL, WFF). For further abbreviations, see legend to Fig. I.

tion around the nuclei in stage 10a (Fig. 2G). During stages $10 \mathrm{~b}$ and 11 , Inx 2 is observed in cytoplasmic clouds and particles (Fig. 2H, I), which become delivered into the growing oocyte during nurse-cell regression [41].

Inx3, which has been shown to heteromerize with Inx2 in embryonic epithelia [40], is colocalized with Inx2 in nurse-cell membranes as well as in lateral, but not in apical, membranes of follicle cells covering the oocyte (Fig. $3 \mathrm{~B}-\mathrm{E})$. This contrasts with its lateral as well as apical appearance in follicle cells covering the nurse cells (Fig. $3 F$ ). In nurse cells, membrane labeling (stage 10, Fig. 3E) and cytoplasmic labeling around the nuclei (stage 7, Fig. 3F) was observed for Inx3.

Inx4 is known to be germ-line specific $[13,37,38]$. We detected Inx4-labelling in the oolemma up to stage 8 and in the nurse-cell membranes up to stage 12 . While continuous labeling was observed between nurse cells, character- istic plaques were found up to stage 9 in the germ-line cell membranes beneath each single follicle cell (Fig. 4A, B; c.f. [13]). In these membrane plaques, Inx4 in germ-line cells appears to be in close vicinity to Inx 2 in follicle cells (Fig. 4D). Inx4-plaques are also present between nurse cells and centripetally migrating follicle cells (stage 10b, Fig. 4C). Beginning with vitellogenesis (stage 8), only Inx2, but not Inx4, was detected in the oolemma (Fig. 4D).

\section{Analysis of dye-coupling following microinjections of innexin antisera}

A critical test for the involvement of Inx2 in intercellular communication between oocyte and follicle cells is the specific blockade of dye-coupling with antibodies directed against cytoplasmic regions of Inx2. We microinjected various antisera in combination with the fluorescent tracer Lucifer Yellow $\mathrm{CH}$ into the oocyte. For technical reasons, the youngest follicles that can be successfully microinjected are of stage 8 [36]. We used stage- 

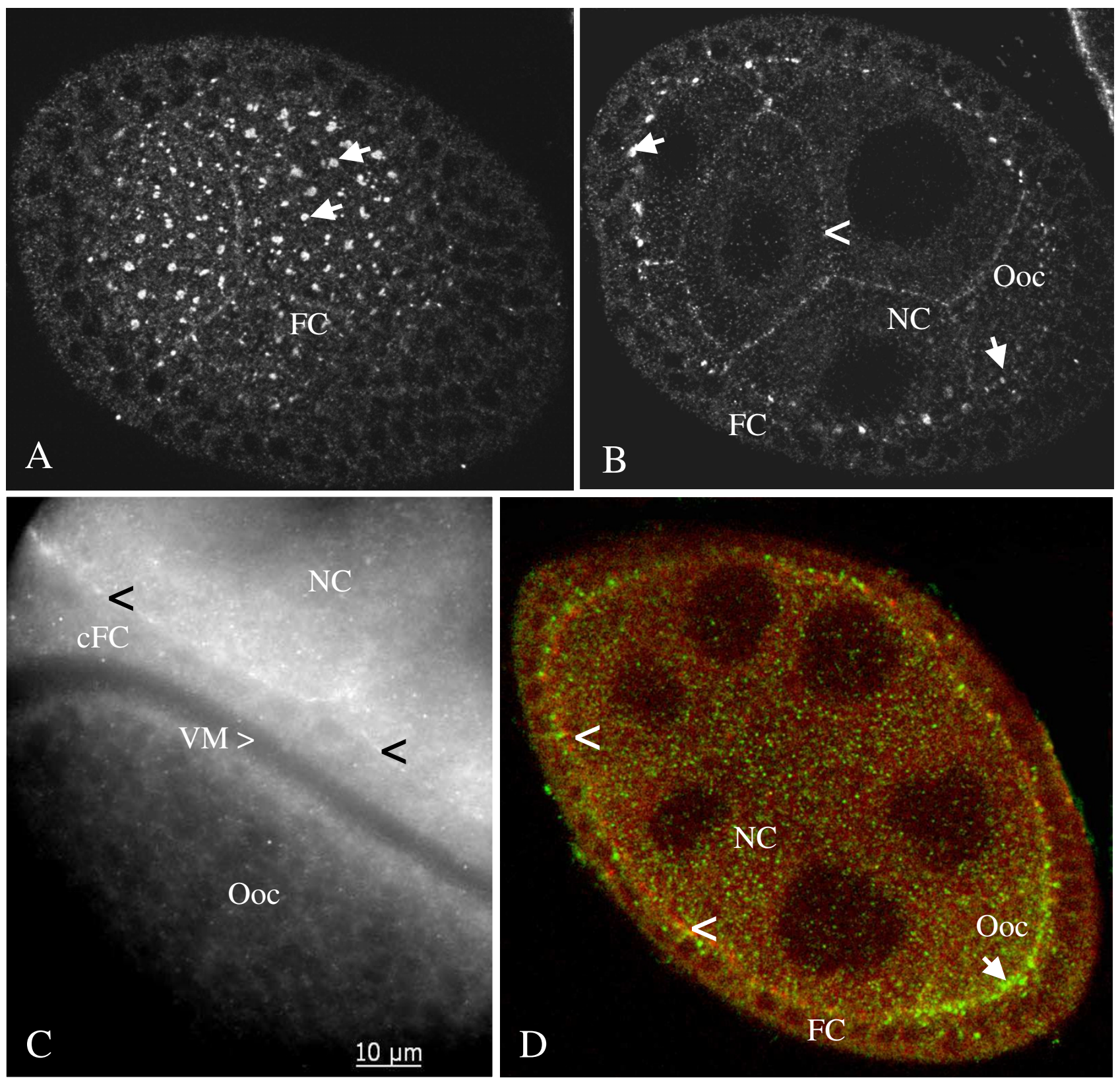

Figure 4

Inx4 is localized to membrane plaques in germ-line cells and seems to interact with Inx2 in follicle cells. A, B: Inx4-plaques (arrows in A (tangential section) and B (median section), stage 6, LSM) were detected between each follicle cell and the underlying oocyte and nurse cells, respectively. Continuous staining was found at the nurse-cell membranes (arrowhead in B). C: Inx4-plaques (black arrowheads, stage IOb, WFF) are located between nurse cells and the centripetally migrating follicle cells. D: Inx2 in follicle-cell membranes (green, Alnx2GP-CL) and Inx4 in nurse-cell membranes (red, Alnx4Rb-CT) are in close vicinity (arrowheads, stage 8, LSM) and seem to interact. Beginning with stage 8, only Inx2 is present between oocyte and follicle cells (arrow). For further abbreviations, see legend to Fig. I.

10 follicles in the present experiment. Since, beginning with stage 8 , only Inx2 was detectable in the oolemma (see above), antisera directed against other innexins served as controls (Table 1).
We found the fraction of follicles showing dye-coupling between oocyte and follicle cells (Fig. 5) largely reduced in comparison to NIS by an antiserum directed against the intracellular C-terminus of Inx2 (AInx2Rb-CT(KLRH), $P<$ 
0.05) and also, but to a minor extent, by an antiserum directed against the intracellular loop of Inx2 (AInx2GP$\mathrm{CL}, P<0.05)$. Antisera directed against either Inx1, Inx3 or Inx4 did not reduce dye-coupling significantly when compared to NIS $(P>0.05$; Table 1$)$. Moreover, only Inx2antisera were found to reduce dye-coupling in a concentration-dependent manner (not shown). These results demonstrate that Inx2 is involved in cell-cell communication by participating in gap-junction hemichannels residing in the oolemma. Although the difference observed between the CT-specific and the CL-specific antiserum was not significant $(P>0.05)$, the data suggest a prominent role of the C-terminus during closure of the pore.

\section{In-vitro development following inhibition of gap-junctional communication}

We further analyzed the developmental capacities of follicles following microinjections of Inx2-antisera into the oocyte. In R-14 medium, stage-10b follicles are able to develop during 8 hours up to stage $14[43,45]$. When compared to NIS, in-vitro development was found to be inhibited in significant fractions of follicles microinjected with either AInx2Rb-CT(KLRH) or AInx2GP-CL $(P<0.05$; Fig. 6). Since Inx4 is present in the oolemma only up to stage 8, AInx4Rb-CT served as a further control. The developmental capacities of follicles microinjected with AInx4Rb-CT were not significantly different from those obtained with NIS $(P>0.05$; Table 2$)$.

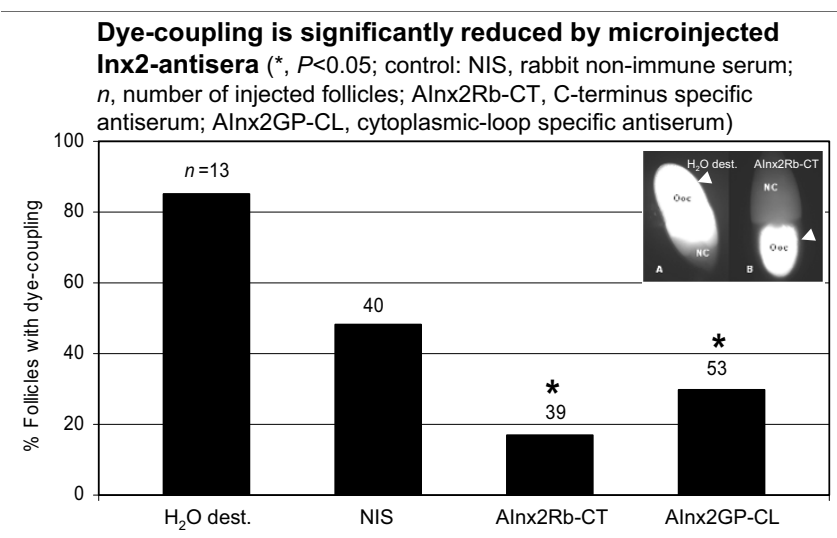

\section{Figure 5}

Microinjected Inx2-antisera inhibit communication between oocyte and follicle cells. In $85 \%\left(\mathrm{H}_{2} \mathrm{O}\right.$ dest.) and $48 \%$ (NIS), respectively, of the control follicles (stage 10), Lucifer Yellow $\mathrm{CH}$ was found to move from the microinjected oocyte $(\mathrm{Ooc})$ into the nurse cells $(\mathrm{NC})$ as well as into the surrounding follicle cells (arrowhead in inset $A$ ). In contrast, in $83 \%$ (Alnx2Rb-CT) and 70\% (Alnx2GP-CL), respectively, of the follicles microinjected with Inx2-antisera, gapjunctional communication between oocyte and follicle cells was blocked (arrowhead in inset B). For further details, see Table I.
Most of the follicles microinjected with Inx2-antisera either stopped their development during stage 10b, which means that processes of follicle-cell differentiation, nursecell regression and oocyte growth were blocked (Fig. 6, inset; c.f. [41]). Or these follicles failed to complete choriogenesis normally, which becomes apparent by missing respiratory appendages, incomplete chorionic layers and sizeable nurse-cell remnants [43]. Therefore, it is obvious that communication between oocyte and follicle cells via Inx2-containing gap-junction channels is essential for normal follicle development through stages 10 to 14 .

\section{Discussion}

Although further proteins have been detected in Drosophila gap junctions [26,31,32], the main invertebrate gap-junction proteins are members of the innexin family $[28,29]$. During oogenesis and embryogenesis, the eight known Drosophila innexins show complex and overlapping expression patterns [37]. In the ovary, the mRNAs of innexins 1, 2, 3, 4 and 7 were found. Innexin mRNAs 1, 2, 3 and (to a minor extent) 7 were observed in the somatic follicle cells, especially in populations of migrating cells. In the oocyte and in the nurse cells, high amounts of innexin mRNAs 2 and 4 were detected, whereas the expression of innexin mRNAs 1, 3 and 7 was found to be rather low [37].

Since the mRNA-expression pattern only gives hints of the distribution and function of a protein, one aim of the

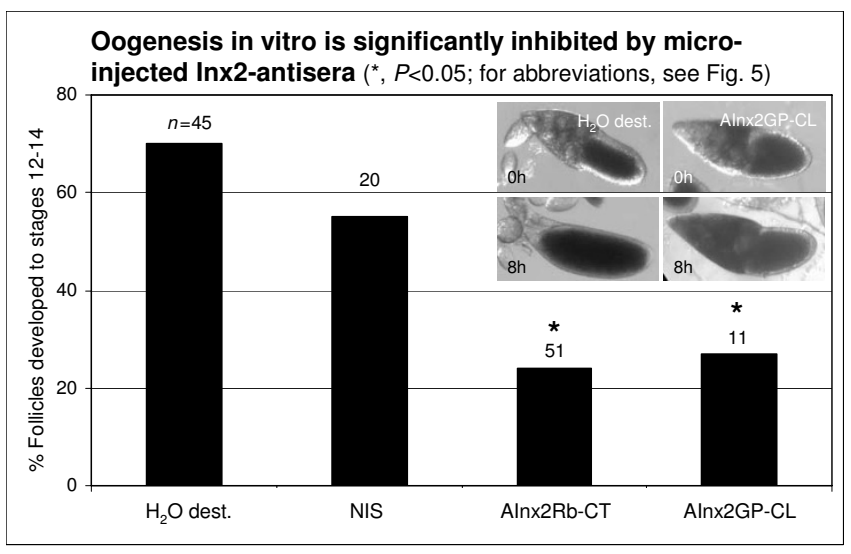

Figure 6

Communication via Inx2-containing channels is essential for normal oogenesis in vitro. Of the microinjected control follicles, $70 \%\left(\mathrm{H}_{2} \mathrm{O}\right.$ dest.) and $55 \%$ (NIS), respectively, developed in $\mathrm{R}-\mathrm{I} 4 \mathrm{~m}$ medium from stage IOb $(0 \mathrm{~h})$ to stages 12-14 (8 h, inset shows example). In contrast, in $76 \%(A \ln \times 2 R b-C T)$ and $73 \%(A \ln \times 2$ GP-CL), respectively, of the follicles microinjected with Inx2-antisera, oogenesis in vitro up to stage 12 was either incomplete or arrested in stage IOb ( $8 \mathrm{~h}$, inset shows example). For further details, see Table 2. 
present study was to reveal the cellular localization of Inx1, 2, 3 and 4 in the ovary. For Inx4, the early phases of localization and functioning have already been addressed: It is germ-line specific and plays an essential role during germ-cell differentiation in the germarium [13,38]. Concerning Inx1, 2 and 3, nothing was known so far. Moreover, it was not clear whether innexins form functional gapjunction channels in the ovary, and whether heteromeric channels (with hemichannels made of different innexins) and/or heterotypic channels (made of different hemichannels) may be involved.

During Drosophila embryogenesis, the roles of Inx1 and Inx2 have been analyzed in detail $[12,14,39,46]$. Both proteins participate in organizing embryonic epithelia by interacting with core proteins of adherens and septate junctions. By various experiments $[40,47]$ it has been shown that Inx2 and Inx3, via their cytoplasmic C-terminal domains, form heterodimers, which are essential for the formation of heteromeric channels and for the integrity and polarity of the embryonic epidermis. Also in a Xenopus-oocyte expression system, Inx2 formed functional heteromeric channels with Inx3, whereas Inx2 alone, but not Inx3 alone, formed homotypic channels [48]. In this system, also Inx1 was found to form heteromeric channels with Inx2 and Inx3, respectively, whereas Inx4 only formed heterotypic channels with Inx2 [28].

Our immunoblot analysis using various innexin anti-peptide sera, that have been characterized before ([13,14,3840] and R. Bauer, pers. com.), revealed the synthesis of Inx1, 2, 3 and 4 in the ovary. Although, in some cases, only weak bands at the calculated molecular masses were detected, further specific bands at lower molecular masses were revealed. Moreover, some antisera appeared to be better suited for immunohistochemistry than for immunoblotting or vice versa. Such phenomena are not unusual. For example, for Inx8 (Shak-B), which is not expressed in the ovary [37], only bands at lower molecular masses than deduced from the amino-acid sequence have been described [9].

Our immunhistochemical analysis revealed that Inx 1 is predominantly localized to the baso-lateral domain of follicle cells, whereas Inx2 is positioned apico-laterally as well as apically between follicle cells and germ-line cells. Inx3 was observed laterally in follicle cells and also in nurse cells, and Inx4 was detected in the oolemma up to stage 8 and in nurse-cell membranes up to stage 12 . While Inx 2 and Inx3 are colocalized between soma cells, Inx2 and Inx4 are colocalized between soma and germ-line cells.

From the analysis of Drosophila mutants, Inx2 has been inferred to exert diverse structural functions $[14,40,49]$.
Presumably, Inx2 is acting indirectly through the formation of membrane channels necessary for intercellular communication. We found that dye-coupling between oocyte and follicle cells was largely reduced by Inx2-antisera directed against both the intracellular C-terminus and the intracellular loop. Analyzing in vitro the developmental capacities of follicles following microinjections of Inx2-antisera revealed defects in follicle-cell differentiation, nurse-cell regression, oocyte growth and choriogenesis. Also in other invertebrates, communication via gap junctions has been found to exert influence on oogenesis [50].

Taken together, our results suggest that Inx1, 2, 3 and 4 are all involved in the formation of gap junctions during oogenesis. Based on immunostaining and microinjection experiments (as well as on heterologous expression $[28,48])$, we propose the existence of a variety of channels between the three cell types: (1) heteromeric channels made of Inx1/Inx3 between follicle cells, (2) homotypic channels made of Inx2 between follicle cells and oocyte, (3) homotypic channels made of Inx2 between germ-line cells, (4) heteromeric channels made of Inx2/Inx3 between follicle cells, (5) heteromeric channels made of Inx2/Inx3 between nurse cells, (6) heterotypic channels made of Inx2/Inx4 between follicle cells and germ-line cells (c.f. [13]), and (7) heterotypic channels made of Inx2/Inx4 between germ-line cells.

In previous studies, using light and electron microscopy, we have shown that antibodies against ductin bind to antigens located in the plasma membranes and in the cytoplasm of various Drosophila tissues, especially of ovarian follicles and embryos [31,32,51,52]. Ductin has been found in both vacuolar-type proton pumps and gap junctions [33]. By microinjection experiments, we have demonstrated that antibodies directed against presumed cytoplasmic regions of ductin block dye-coupling between germ-line and soma cells and exert influence on oogenesis as well as on embryogenesis [31,32].

\section{Conclusion}

Thus, antibodies against both Inx2 and ductin inhibit gapjunctional communication between oocyte and follicle cells in Drosophila stage-10 follicles, and both proteins are present in the oolemma during this stage. In both cases, microinjected antibodies essentially affected normal follicle development. It remains to be clarified how innexins and ductins in gap junctions are related, and which molecules are to be exchanged between soma and germ-line cells.

\section{Abbreviations}

AInx: anti-innexin; BSA: bovine serum albumine; CL: cytoplasmic loop; CT: C-terminus; DAPI: 4',6-diamidino- 
2-phenylindole; GP: guinea pig; Inx: innexin; LSM: laserscanning microscope; LY: Lucifer Yellow CH; NIS: nonimmune serum; PBS: phosphate buffered saline; Rb: rabbit; SDS-PAGE: sodium dodecyl sulphate polyacrylamide gel-electrophoresis; WFF: wide-field fluorescence.

\section{Authors' contributions}

JB conceived the study, reviewed and analyzed the data and wrote the manuscript. JZ carried out the experiments under the supervision of JB and was involved in data analysis. Both authors read and approved the final manuscript.

\section{Acknowledgements}

We are indebted to Reinhard Bauer and Michael Hoch (Bonn, Germany) for providing the antisera against innexins I, 2 and 3, to Sally Tazuke (Philadelphia, PA, USA) for the gift of the innexin- 4 antiserum, and to Maria Bugaro and Andrzej Steckiewicz for technical assistance. Financial support by RWTH Aachen University is also acknowledged.

\section{References}

I. Spradling A: Developmental genetics of oogenesis. In The Development of Drosophila melanogaster Edited by: Bate M, Martinez Arias A. Cold Spring Harbor, New York: Cold Spring Harbor Laboratory Press; 1993:1-70.

2. Bownes M: Interactions between germ cells and somatic cells in Drosophila melanogaster. Semin Dev Biol 1994, 5:3 I-42.

3. Horne-Badovinac S, Bilder D: Mass transit: Epithelial morphogenesis in the Drosophila egg chamber. Dev Dyn 2005 232:559-574.

4. Schüpbach T, Clifford RJ, Manseau LJ, Price JV: Dorsoventral signaling processes in Drosophila oogenesis. In Cell-Cell Interactions in Early Development Edited by: Gerhart J. New York: Wiley-Liss; 1991:163-174.

5. Stevens LM, Nüsslein-Volhard C: Development of the terminal anlagen of the Drosophila embryo depends upon interactions between the germline and the somatic follicle cells. In Cell-Cell Interactions in Early Development Edited by: Gerhart J. New York: Wiley-Liss; | 1991:145-162.

6. Riechmann V, Ephrussi A: Axis formation during Drosophila oogenesis. Curr Opin Genet Dev 200I, I I:374-383.

7. Jursnich VA, Fraser SE, Held LI, Ryerse JS, Bryant PJ: Defective gapjunctional communication associated with imaginal disc over-growth and degeneration caused by mutations of the dco gene in Drosophila. Dev Biol 1990, 140:413-429.

8. Sun YA, Wyman RJ: Passover eliminates gap junctional communication between neurons of the giant fiber system in Drosophila. J Neurobiol 1996, 30:340-348.

9. Phelan P, Stebbings LA, Baines RA, Bacon JP, Davies JA, Ford C: Drosophila Shaking-B protein forms gap junctions in paired Xenopus oocytes. Nature 1998, 391:18I-184.

10. Todman MG, Baines RA, Stebbings LA, Davies JA, Bacon JP: Gapjunctional communication between developing Drosophila muscles is essential for their normal development. Dev Genet 1999, 24:57-68.

1I. Curtin KD, Zhang Z, Wyman RI: Gap junction proteins are not interchangeable in development of neural function in the Drosophila visual system. J Cell Sci 2002, I I 5:3379-3388.

12. Bauer R, Lehmann C, Fuss B, Eckardt F, Hoch M: The Drosophila gap junction channel gene innexin 2 controls foregut development in response to Wingless signalling. J Cell Sci 2002, I I5:1859-1867.

13. Tazuke SI, Schulz C, Gilboa L, Fogarty M, Mahowald AP, Guichet A, Ephrussi A, Wood CG, Lehmann R, Fuller MT: A germline-specific gap junction protein required for survival of differentiating early germ cells. Development 2002, 129:2529-2539.

14. Bauer R, Lehmann C, Martini J, Eckardt F, Hoch M: Gap junction channel protein innexin $\mathbf{2}$ is essential for epithelial morpho- genesis in the Drosophila embryo. Mol Biol Cell 2004, 15:2992-3004.

15. Caveney S: The role of gap junctions in development. Annu Rev Physiol 1985, 47:319-335.

16. Warner AE: Gap junctions in development - a perspective. Semin Cell Biol 1992, 3:8I-91.

17. White TW: Nonredundant gap junction function. News Physiol Sci 2003, 1 8:95-99.

18. Wei Cl, $\mathrm{Xu} X$, Lo CW: Connexins and cell signaling in development and disease. Annu Rev Cell Dev Biol 2004, 20:8I I-838.

19. Levin M: Gap junctional communication in morphogenesis. Prog Biophys Mol Biol 2007, 94(I-2): I86-206.

20. Berdan RC: Intercellular communication in arthropods: biophysical, ultrastructural, and biochemical approaches. In Cellto-Cell Communication Edited by: De Mello WC. New York, London: Plenum Press; 1987:299-370.

21. Waksmonski SL, Woodruff RI: For uptake of yolk precursors, epithelial cell-oocyte gap junctional communication is required by insects representing six different orders. J Insect Physiol 2002, 48:667-675.

22. Brooks RA, Woodruff RI: Calmodulin transmitted through gap junctions stimulates endocytic incorporation of yolk precursors in insect oocytes. Dev Biol 2004, 27 I:339-349.

23. Wolvetang EJ, Pera MF, Zuckerman KS: Gap junction mediated transport of shRNA between embryonic stem cells. Biochem Biophys Res Commun 2007, 363(3):610-6I5.

24. White TW, Paul DL: Genetic diseases and gene knockouts reveal diverse connexin functions. Annu Rev Physiol 1999, 61:283-310.

25. Cruciani $\mathrm{V}$, Mikalsen SO: The vertebrate connexin family. Cell Mol Life Sci 2006, 63: I I 25- I I 40.

26. Phelan P: Gap junction communication in invertebrates: The innexin gene family. Curr Top Membranes 2000, 49:389-422.

27. Phelan P, Starich TA: Innexins get into the gap. BioEssays 200I, 23:388-396.

28. Phelan P: Innexins: members of an evolutionarily conserved family of gap-junction proteins. Biochim Biophys Acta 2005, I 7 I I (2):225-245.

29. Bauer R, Löer B, Ostrowski K, Martini J, Weimbs A, Lechner H, Hoch M: Intercellular communication: the Drosophila innexin multiprotein family of gap junction proteins. Chemistry \& Biology 2005, 12:515-526.

30. Barbe MT, Monyer H, Bruzzone R: Cell-cell communication beyond connexins: the pannexin channels. Physiology 2006, 21:103-114.

31. Bohrmann J: Antisera against a channel-forming 16 kDa protein inhibit dye-coupling and bind to cell membranes in Drosophila ovarian follicles. J Cell Sci 1993, 105:5 I3-5 I8.

32. Bohrmann J, Lämmel $\mathrm{H}$ : Microinjected antisera against ductin affect gastrulation in Drosophila melanogaster. Int I Dev Bio 1998, 42:709-72।.

33. Finbow ME, Pitts JD: Structure of the ductin channel. Biosci Rep 1998, 18:287-297.

34. Mahowald AP: Ultrastructural observations on oogenesis in Drosophila. J Morphol 1972, 137:29-48.

35. Giorgi F, Postlethwait JH: Development of gap junctions in normal and mutant ovaries of Drosophila melanogaster. J Morphol 1985, 185: II5-129.

36. Bohrmann J, Haas-Assenbaum A: Gap junctions in ovarian follicles of Drosophila melanogaster: inhibition and promotion of dye-coupling between oocyte and follicle cells. Cell Tissue Res 1993, 273:163-173.

37. Stebbings LA, Todman MG, Phillipps R, Greer CE, Tam J, Phelan P, Jacobs K, Bacon JP, Davies JA: Gap junctions in Drosophila: developmental expression of the entire innexin gene family. Mech Dev 2002, I I 3:197-205.

38. Gilboa L, Forbes A, Tazuke SI, Fuller MT, Lehmann R: Germ line stem cell differentiation in Drosophila requires gap junctions and proceeds via an intermediate state. Development 2003, I30:6625-6634.

39. Bauer R, Martini J, Lehmann C, Hoch M: Cellular distribution of innexin $I$ and 2 gap junctional channel proteins in epithelia of the Drosophila embryo. Cell Commun Adhes 2003, 10:221-225.

40. Lehmann C, Lechner H, Löer B, Knieps M, Herrmann S, Famulok M, Bauer R, Hoch M: Heteromerization of innexin gap junction 
proteins regulates epithelial tissue organization in Drosophila. Mol Biol Cell 2006, 17:1676-1685.

41. Mahowald AP, Kambysellis MP: Oogenesis. In Genetics and Biology of Drosophila Volume 2d. Edited by: Ashburner M, Wright TRF. New York: Academic Press; 1980:141-224.

42. Robb JA: Maintenance of imaginal discs of Drosophila melanogaster in chemically defined media. J Cell Biol 1969, 41:876-885.

43. Bohrmann J: In vitro culture of Drosophila ovarian follicles: the influence of different media on development, RNA synthesis, protein synthesis and potassium uptake. Roux's Arch Dev Biol 1991, 199:315-326.

44. Bohrmann J, Schill S: Cytoplasmic transport in Drosophila ovarian follicles: the migration of microinjected fluorescent probes through intercellular bridges depends neither on electrical charge nor on external osmolarity. Int J Dev Biol 1997, 41:499-507.

45. Bohrmann J, Sander K: Aberrant oogenesis in the patterning mutant dicephalic of Drosophila melanogaster: time-lapse recordings and volumetry in vitro. Roux's Arch Dev Biol 1987, 196:279-285.

46. Bauer $\mathrm{R}$, Weimbs $A$, Lechner $H$, Hoch M: DE-cadherin, a core component of the adherens junction complex modifies subcellular localization of the Drosophila gap junction protein innexin 2. Cell Commun Adhes 2006, 13:103-I 14.

47. Knieps M, Herrmann S, Lehmann C, Löer B, Hoch M, Famulok M: Anti-innexin 2 aptamers specifically inhibit the heterologous interaction of the innexin 2 and innexin 3 carboxyl-termini in vitro. Biol Chem 2007, 388:56I-568.

48. Stebbings LA, Todman MG, Phelan P, Bacon JP, Davies JA: Two Drosophila innexins are expressed in overlapping domains and cooperate to form gap-junction channels. Mol Biol Cell 2000, I I:2459-2470.

49. Tazuke SI, Chan M: Dosophila Innexin-2 is required in the ovary for follicle cell migration and nurse cell dumping. International Gap Junction Conference, Canada; 2005:79.

50. Whitten SJ, Miller MA: The role of gap junctions in Caenorhabditis elegans oocyte maturation and fertilization. Dev Biol 2007, 301:432-446.

5I. Bohrmann J, Braun B: Na,K-ATPase and V-ATPase in ovarian follicles of Drosophila melanogaster. Biol Cell 1999, 9 I:85-98.

52. Bohrmann J, Bonafede $\mathrm{A}$ : Tissue-specific distribution and variation of the channel-forming protein ductin during development of Drosophila melanogaster. Int J Dev Biol 2000, 44:883-890.
Publish with Bio Med Central and every scientist can read your work free of charge

"BioMed Central will be the most significant development for disseminating the results of biomedical research in our lifetime. "

Sir Paul Nurse, Cancer Research UK

Your research papers will be:

- available free of charge to the entire biomedical community

- peer reviewed and published immediately upon acceptance

- cited in PubMed and archived on PubMed Central

- yours - you keep the copyright

Submit your manuscript here:

http://www.biomedcentral.com/info/publishing_adv.asp
BioMedcentral 